\title{
Managing buccal space tumours
}

\section{Dear Editor,}

The buccal space is an infrequently addressed fascial space of the head and neck region. Intrinsic tumours of the buccal space are rare and hence present a management challenge. In a recent systematic review of 217 patients, 4 main surgical approaches were identified (intraoral, extended parotidectomy/rhytidectomy, transcutaneous and paranasal lip-split, and upper or lower cheek flap). ${ }^{1,2}$ We present a side-by-side comparison demonstrating factors that contribute to the choice of surgical approach, their pros and cons, and their respective treatment outcomes. Through this case series, we aim to offer a guide to surgical planning for intrinsic buccal space tumours by proposing a novel management algorithm. This study is approved by the Tan Tock Seng Hospital's ethics board (DSRB Ref: 2020/00803).

Case series illustrating different surgical approaches. Over the course of 10 years, we managed 5 buccal space lesions at our ear, nose and throat department. Three cases were excised via the transoral approach, one via an extended parotidectomy approach, and one via an upper cheek flap approach.

The 3 buccal lesions excised via a transoral approach consisted of a 76-year-old man with a $5 \times 6 \mathrm{~cm}$ facial nerve schwannoma within the buccal space; a 29 -yearold woman with a $2 \times 2 \mathrm{~cm}$ buccal space pleomorphic adenoma and a 56 -year-old man with a $1.5 \times 2 \mathrm{~cm}$ palpable buccal space lymph node. The transoral approach was chosen for these patients based on physical examination and imaging findings. A characteristic finding was that these lesions were seen to bulge medially towards the buccal mucosa. Postoperatively, the first patient had a temporary palsy of the buccal branch of facial nerve that resolved in 6 months, while the second patient experienced a sialocele that resolved with serial aspirations. These 3 cases were followed up for a duration of 8 to 41 months and demonstrated no recurrence.

The buccal lesion excised via the extended parotidectomy approach was a 55-year-old man with a $1.5 \times 1.5 \mathrm{~cm}$ high grade polymorphous adenocarcinoma of the buccal space. Prior to surgery, blood was expressed from the Stensen's duct on applying direct pressure to this cheek lump, raising concerns of a malignancy. On examination, this patient had a bulge on the external cheek. The preoperative computed tomography (CT) scan showed a buccal space tumour separate from the parotid gland. Intraoperatively, it was attached to an accessory parotid duct. The zygomatic and buccal branches of the facial nerve were identified and preserved utilising a facial nerve monitor. ${ }^{3}$ Postoperatively he experienced a sialocele that resolved with conservative management. He had no facial weakness. He underwent radiotherapy and remained recurrence-free for 10 years.

The buccal lesion excised via the upper cheek flap approach was a 48 -year-old man with a $4.5 \times 4 \mathrm{~cm}$ left buccal pleomorphic adenoma. On CT, this tumour was centred anteromedial to the left masseter muscle, extending into the retro-maxillary region and bulged towards the buccal mucosa. The external incision was connected with a circumferential incision around the involved buccal mucosa and the tumour was removed en bloc with clear margins. The defect was reconstructed with a radial forearm free-flap. Postoperatively he had an episode of chin abscess and required additional surgical flap debulking. He remained recurrence-free for 10 years.

Discussion. In 1945, Kostrubala described the buccal space as a potential space between the buccinator muscle and the more superficial muscles of facial expression. ${ }^{4}$ Head and neck surgeons commonly encounter oral cavity squamous cell carcinoma invading into the buccal space, and the oncological management of these lesions has been described and discussed in large international studies. ${ }^{5-7}$ Our paper, however, discusses intrinsic buccal space lesions that are distinct from lesions arising from the skin of the cheek, buccal mucosa or other adjacent spaces. ${ }^{8}$ These tumours are rare, and based on a recent systematic review, the most common lesions of the buccal space were vascular $(29.4 \%)$ and salivary gland $(27.1 \%)$ in origin, followed by a wide variety of soft tissue tumours. ${ }^{1}$ Majority of vascular lesions were buccal space haemangiomas $(n=50)$, but these were not encountered at our centre likely due to early diagnosis, treatment and resolution of disease in the local paediatric population. The commonest tumour of salivary gland origin was pleomorphic adenoma, but a significant proportion were malignant tumours such as adenoid cystic carcinoma, acinic cell carcinoma and mucoepidermoid carcinoma.

The first 3 cases demonstrate how a transoral approach can be used to successfully excise buccal space tumours. We found that a mobile lesion with a bulge intraorally is 


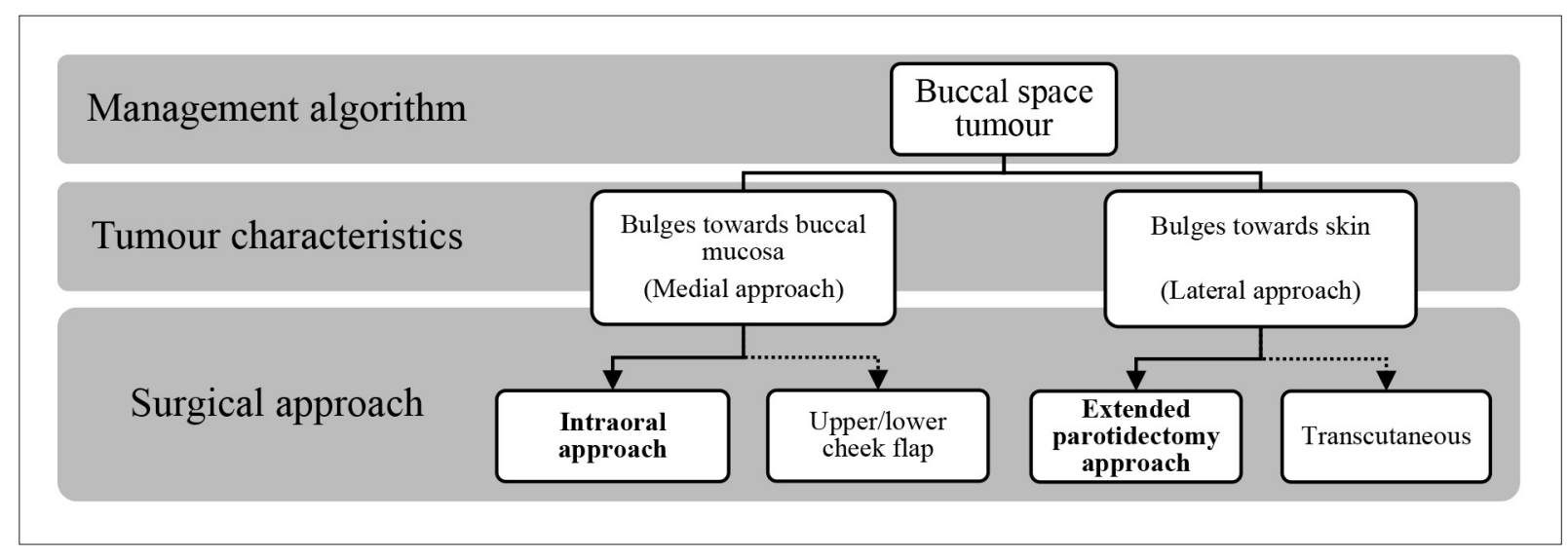

Fig. 1. Management algorithm of buccal space tumours based on tumour characteristics.

often amenable to this approach. This was even possible for a $5 \times 6 \mathrm{~cm}$ large mass with blunt finger dissection. The transoral approach avoids a cutaneous scar, however has inferior visualisation of facial nerve branches and the Stensen's duct. While the buccal branches are known to have rich cross-innervation and redundancy, there is still a higher theoretical risk of facial weakness and sialocele formation. ${ }^{9,10}$

The extended parotidectomy approach involves a modified Blair's incision extended superiorly and inferiorly. A flap is raised anteriorly on a broad front. The buccal branches of the facial nerve and the Stensen's duct are identified anterior to the parotid gland, and careful dissection towards the tumour allows for its safe excision. The accessory parotid duct in Case 4 could be isolated and ligated. The rhytidectomy approach uses a similar technique; while it requires more extensive dissection, it facilitates hiding the scar behind the hairline and reconstruction by superficial musculoaponeurotic system interposition. In general, these approaches involve a larger incision while maintaining acceptable cosmesis and provides superior visualisation of the facial nerve and Stensen's duct.

The transcutaneous approach to the buccal space is not encouraged as it results in an obvious scar over the cheek and provides inadequate visualisation of structures. The upper/lower cheek flap approach with free flap reconstruction can be used for specific cases. This approach was chosen for Case 5 which involved a large buccal space tumour adherent to the buccal mucosa. Additional concerns include the challenging task of retrograde dissection of the facial nerve and the capability of free-flap reconstruction.

With the aforementioned list of surgical options and considerations available, deciding on the ideal approach can be challenging. Hence, we propose a novel management algorithm to help with this decision (Fig. 1). In our experience, the tumour's characteristics is the chief consideration in determining an approach. When the buccal lesion bulges towards the buccal mucosa, a medial approach is recommended; when it bulges towards the skin, a lateral approach is preferred. Pertaining to the medial approach, benign tumours that are freely mobile with distinct margins are amenable to transoral excision; more extensive, infiltrative or malignant tumours may require the upper/lower cheek flap approach. With regard to the lateral approach, the extended parotidectomy approach is recommended over the transcutaneous approach because of the aforementioned reasons. While CT and magnetic resonance imaging scans are useful in demonstrating the location and extent of the mass, they have been found to be limited in differentiating benign and malignant salivary gland tumours. ${ }^{11,12}$ This is because they often present early as a small, distinct and mobile lump in the cheek. For such cases, fine needle aspiration cytology may be helpful. ${ }^{13}$ When dealing with malignant and infiltrative lesions, adequate exposure to obtain clear oncologic margins is imperative, and this can be done by either the upper/ lower cheek flap or extended parotidectomy approach depending on the direction of bulge of the buccal space tumour. On rare occasions, where the buccal space tumour invades into the adjacent masticator or intratemporal spaces, this too should be taken into account for surgical planning. On reviewing the medical literature of buccal space tumours, we found that vast majority of buccal space tumours would be amenable to either the transoral or extended parotidectomy approach. ${ }^{1,11,12,14}$

This case series illustrates the role of different surgical approaches to the buccal space. The transoral 
and extended parotidectomy approaches are the principal surgical approaches, both of which are essential for their respective clinical scenarios. We propose a novel management algorithm to determine the recommended surgical approach for these rare tumours.

\section{REFERENCES}

1. Hasan Z, Tan D, Buchanan M, et al. Buccal space tumours. Auris Nasus Larynx 2019;46:160-6.

2. Riffat $F$, Hasan $Z$, Buchanan $M$, et al. A diagnostic and surgical approach to masses of the buccal space: a case series. Aust J Otolaryngol 2019;2:18.

3. Sood AJ, Houlton JJ, Nguyen SA, et al. Facial nerve monitoring during parotidectomy: a systematic review and meta-analysis. Otolaryngol Head Neck Surg 2015;152:631-7.

4. Kostrubala JG. Potential anatomical spaces in the face. Am J Surg 1945;68:28-37.

5. Pfister DG, Spencer S, Adelstein D, et al. Head and Neck Cancers, Version 2.2020, NCCN Clinical Practice Guidelines in Oncology. J Natl Compr Canc Netw 2020;18:873-98.

6. Lim AA, Wee TH, Wong RC. Epidemiology of oral cancer diagnosed at a Singapore tertiary healthcare institution. Ann Acad Med Singap 2014;43:96-101.

7. Kuek LB, Chin TW, Fong KW. Lip and intraoral tumours: a local perspective. Ann Acad Med Singap 1990;19:178-81.

8. Phillip Huyett, Eugene N Myers. Mass in the Buccal Space. In: Eugene N Myers, Carl H Snyderman (Eds.) Operative Otorhinolaryngology (pp.601-6). 3rd ed. Elsevier Ltd; 2018.
9. Dhiwakar M, Khan ZA. Sacrificing the buccal branch of the facial nerve during parotidectomy. Head Neck 2016;38:1821-5.

10. Van Sickels, Alexander JM. Parotid duct injuries. Oral Surg Oral Med Oral Pathol 1981;52:364-7.

11. Kurabayashi T, Ida M, Yoshino N, et al. Computed tomography in the diagnosis of buccal space masses. Dentomaxillofac Radiol 1997;26:347-53.

12. Kurabayashi $T$, Ida $M$, Tetsumura $A$, et al. MR imaging of benign and malignant lesions in the buccal space. Dentomaxillofac Radiol 2002;31:344-9.

13. Choy KCC, Bundele MM, Li H, et al. Risk stratification of fine-needle aspiration cytology of parotid neoplasms based on the Milan system-Experience from a tertiary center in Asia. Head Neck 2019;41:3125-32.

14. Sharma A, Deshmukh S, Shaikh A, et al. Pleomorphic adenoma of the minor salivary gland of the cheek. Singapore Med J 2013;54:e183-4.

Augustine Yui Ler Chai ${ }^{1}$ MRCS, Ming Yann Lim ${ }^{1}{ }_{F A M S}(O R L)$,

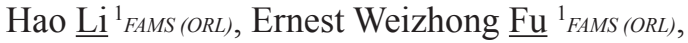
Jin Keat Siow ${ }^{1}$ FAMS (ORL), Yijin Jereme Gan ${ }^{1}$ FAMS (ORL) ${ }^{1}$ Department of Otorhinolaryngology, Tan Tock Seng Hospital,
Singapore

Correspondence: Dr Gan Yijin Jereme, Department of Otorhinolaryngology, Tan Tock Seng Hospital, 11 Jln Tan Tock Seng, Singapore 308433.

Email: jereme_yj_gan@ttsh.com.sg 